\section{¡Sedentarismo e inactividad física no son lo mismo!: una actualización de conceptos orientada a la prescripción del ejercicio físico para la salud}

\section{Sedentary behaviour and physical inactivity is not the same!: An update of concepts oriented towards the prescription of physical exercise for health}

\section{Sr. Editor:}

En el año 2010, la Organización Mundial de la Salud estimó que 3,2 millones de personas mueren cada año en el mundo debido a "inactividad física", convirtiéndose en el cuarto factor de riesgo más importante asociado a mortalidad ${ }^{1}$. No obstante, durante la última década el "sedentarismo" se ha convertido en otro potente factor de riesgo asociado a enfermedades crónicas no transmisibles y mortalidad" ${ }^{1-3}$. Si bien ambos conceptos ("inactividad física" y "sedentarismo") se relacionan ${ }^{4}$, es crucial entender que no son lo mismo ${ }^{1}$, y que ambos actúan de forma independiente sobre nuestra salud 5 .

La conducta sedentaria es definida como la carencia de movimiento durante las horas de vigilia a lo largo del dia ${ }^{1}$, y es caracterizada por actividades que sobrepasan levemente el gasto energético basal ( 1 MET), como: ver televisión, estar acostado o sentado ${ }^{3,4}$. Las actividades sedentarias son la primera categoría de un continuum de clasificación de actividad física (AF) basado en la intensidad de esta: 1) Actividades de tipo sedentarias ( 1 a 1,5 MET); 2) AF ligera (1,5 a 2,9 MET); 3 ) AF moderada (3 a 5,9 MET); y 4) AF vigorosa ( $\geq 6 \mathrm{MET}$ ).

A su vez, la inactividad física se define como el no cumplimiento de las recomendaciones mínimas internacionales de AF para la salud de la población $(\geq 150 \mathrm{~min}$ de actividad física de intensidad moderada o vigorosa por semana, o bien, lograr un gasto energético $\geq 600 \mathrm{MET} / \mathrm{min} /$ semana $)^{4}$. Personas cuya AF esté por debajo de estas recomendaciones se consideran "inactivas

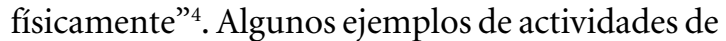
intensidad moderada o vigorosa son: subir escaleras, correr, andar en bicicleta, nadar, etc. No obstante, estas recomendaciones de AF no incluyen actividades de intensidad ligera, como: ordenar o limpiar la casa, lavar los platos, planchar, cocinar, algunas tareas de oficina, etc., las cuales se realizan cotidianamente y también contribuirían a la salud de las personas 4 .

Por ende, es importante que el sedentarismo no se confunda con la inactividad física, ya que una persona puede cumplir con las recomendaciones de AF, pero al mismo tiempo puede destinar la mayor parte del día a actividades de tipo sedentarias ${ }^{2-5}$. Un claro ejemplo de esto es el trabajador que realiza $40 \mathrm{~min}$ de actividad física moderada-vigorosa diariamente, pero acumula cerca de $15 \mathrm{~h}$ diarias entre estar sentado frente al computador, conduciendo al trabajo, descansando viendo televisión, etc.

Esto último es una característica emergente de la población chilena, la cual destina en promedio $64 \%$ del tiempo diario a actividades sedentarias, $29 \%$ a AF ligera, $4 \%$ a AF moderada y $3 \%$ a AF vigorosa $^{6}$. Cabe señalar que, en Chile, $31 \%$ de la población no es físicamente activa o realiza $<150$ min a la semana de AF de intensidad moderada a vigorosa ${ }^{6}$.

Futuras iniciativas y campañas orientadas a incrementar los niveles de AF poblacional deberían considerar las diferencias entre los conceptos aquí discutidos, de manera de poder orientar sus esfuerzos correctamente, ya que los beneficios atribuidos a cumplir con las recomendaciones de AF podrían reducirse considerablemente si la persona destina la mayor parte de sus horas de vigilia a realizar actividades sedentarias ${ }^{2-5}$.

Carlos Cristi-Montero ${ }^{1 a}$, Carlos Celis-Morales ${ }^{2,3 b}$, Rodrigo Ramírez-Campillo ${ }^{4 c}$, Nicolás Aguilar-Farías ${ }^{5 d}$, Cristian Álvarez ${ }^{6 e}$, Fernando Rodríguez-Rodríguez ${ }^{1 f}$

${ }^{1}$ Grupo IRyS, Escuela de Educación Física, Pontificia Universidad Católica de Valparaíso. Valparaíso, Chile.

${ }^{2}$ BHF Glasgow Cardiovascular Research Centre, Institute of Cardiovascular and Medical Science, Glasgow University. Glasgow, United Kingdom.

${ }^{3}$ Human Nutrition Research Centre, Institute of Cellular Medicine, Newcastle University. Newcastle upon Tyne, United Kingdom.

${ }^{4}$ Departamento de Ciencias de la Actividad Física, Universidad de Los Lagos. Osorno, Chile. ${ }^{5}$ Departamento de Educación Física, Deportes y Recreación, Universidad de La Frontera. Temuco, Chile. 


\author{
${ }^{6}$ Centro de Salud Familiar Tomas Rojas. \\ Región de Los Ríos, Chile. \\ aProfesor de Educación Física, Doctor en \\ Ciencias de la Actividad Física y Deportes. \\ ${ }^{b}$ Profesor de Educación Física, Doctor en \\ Ciencias Cardiovasculares y Biomédicas. \\ 'Profesor de Educación Física, \\ Doctor en Ciencias de la Salud. \\ ${ }^{d}$ Kinesiólogo, Master en Fisiología \\ Clínica del Ejercicio. \\ eProfesor de Educación Física, \\ Master en Entrenamiento Deportivo. \\ ${ }^{f}$ Profesor de Educación Física, Doctor \\ en Ciencias del Ejercicio, implicadas en el \\ Rendimiento Físico del ser humano.
}

\section{Referencias}

1. World Health Organization. Global health risks: mortality and burden of disease attributable to selected major risks. Geneva: World Health Organization; 2009; 11.

2. Chau JY, Grunseit AC, Chey T, Stamatakis E, Brown WJ, Matthews CE, et al. Daily Sitting Time and All-Cause Mortality: A Meta-Analysis. Plos One 2013; 8 (11): 14.

3. Tremblay MS, Colley RC, Saunders TJ, Healy GN, Owen
N. Physiological and health implications of a sedentary lifestyle. Appl Physiol Nutr Metab 2010; 35 (6): 725-40.

4. Cristi-Montero C, Rodríguez FR. [The paradox of being physically active but sedentary or sedentary but physically active]. Rev Med Chile 2014; 142 (1): 72-8.

5. Kulinski JP, Khera A, Ayers CR, Das SR, de Lemos JA, Blair SN, et al. Association between cardiorespiratory fitness and accelerometer-derived physical activity and sedentary time in the general population. Mayo Clin Proc 2014; 89 (8): 1063-71.

6. Celis-Morales CA, Pérez-Bravo F, Ibañes L, Sanzana $\mathrm{R}$, Hormázabal E, Ulloa N, et al. Insulin Resistance in Chileans of European and Indigenous Descent: Evidence for an Ethnicity x Environment Interaction. PLoS ONE 2011; 6 (9): e24690.

Fuentes de financiamiento: Estudio autofinanciado. Conflictos de intereses: Ninguno que declarar.

Correspondencia a:

Dr. Carlos Cristi-Montero.

Grupo IRyS. Escuela de Educación Física. Pontificia Universidad

Católica de Valparaíso. Dirección: Av. El Bosque 1290.

Viña del Mar, Chile.

carlos.cristi.montero@gmail.com 